\title{
Closed-Form Prediction of the Thermal and Structural Response of a Perimeter Column in a Fire
}

\author{
Spencer E. Quiel ${ }^{1, *}$ and Maria E. M. Garlock ${ }^{2}$ \\ ${ }^{1}$ Hinman Consulting Engineers Alexandria, VA, USA, ${ }^{2}$ Department of Civil and Environmental Engineering, Princeton \\ University, Princeton, NJ, USA
}

\begin{abstract}
This paper proposes a simplified closed-form methodology with which to predict the thermal and structural response of steel perimeter columns in high-rise building frames exposed to fire. Due to their orientation in the building compartment, perimeter columns are heated on three sides and will develop a thermal gradient through their crosssectional depth. Restraint of the thermal expansion associated with this gradient will cause these members to experience a combination of axial load $(P)$ and bending moment $(M)$, thus acting as beam-columns. At high temperatures, the thrudepth gradient will alter the plastic capacity and mechanical behavior of the perimeter column, leading to plastic $P-M$ behavior that is not captured under the assumption of uniform cross-sectional temperature. Simplified methodologies are proposed to calculate the following: (1) the thru-depth temperature distribution that develops due to three-sided heating, (2) the gradient-induced changes in plastic capacity, and (3) the gradient-induced changes in demand (i.e. $P$ and $M$ ). These methodologies are sufficiently simple for use in code-based design and can be implemented via a spreadsheet because they are closed-form. The individual results of each simple methodology as well as their combination are validated against the results of computational thermal and structural analysis, showing good agreement.
\end{abstract}

Keywords: Fire, beam-column, steel, thermal gradient, performance-based analysis.

\section{INTRODUCTION}

Due to the restraint of thermal expansion, many members in a fire-exposed steel building frame will experience a combination of axial load $(P)$ and bending moment $(M)$, thus acting as beam-columns. In particular, members that develop thermal gradients through their depth due to their orientation in the building frame will experience a combination of $P$ and $M$ as they encounter restraint to both axial thermal expansion (due to an overall temperature increase) and thermal bowing (due to the thermal gradient) in addition to their gravity loads. The authors have described in detail the changes in plastic $P-M$ capacity [1] and $P-M$ response [2] experienced by these members, which include perimeter columns (exposed to fire on three sides) and floor beams (whose top face is shielded by the slab). A collaborative experimental study between researchers at Michigan State University and Princeton University successfully demonstrated these behaviors for members loaded in a furnace that simulated the performance of perimeter columns [3]. A resulting numerical study validated the ability of computational models to predict the experimental changes in $P-M$ caused by hightemperature thru-depth thermal gradients [3]. This paper addresses the need for performance-based tools to calculate the performance of elements that act as beam-columns and develop thermal gradients through their depth due to fire exposure. Simplified methods are proposed to predict the thru-depth temperature distribution, capacity, and demand induced in beam-columns that are non-uniformly heated.

*Address correspondence to this author at Hinman Consulting Engineers Alexandria, VA, USA; Tel: 703-416-6780; Fax: 703-836-4423; E-mail: squiel@hce.com;
As shown in Fig. (1), performance-based structural-fire analysis is accomplished in two uncoupled phases once a temperature-time relationship representing the fire is selected or calculated: thermal analysis followed by structural analysis. The analyst can select either computational finite element (FE) analysis or closed-form simplified methods to perform these analyses, each having advantages and disadvantages. Computational tools provide a higher level of calculation detail and precision but at a higher cost. Simplified methods can be used more efficiently, but they rely on simplifying assumptions and may not provide the same level of accuracy as computational solutions. Simplified tools that can predict the response of fire-exposed structural members with similar precision as computational methods allows the analyst reap the benefits of using simplified methods without sacrificing accuracy.

In this paper, a simplified methodology is proposed, based on the studies discussed in the aforementioned papers, which explicitly calculates the response of fire-exposed steel beam-columns that develop a thru-depth thermal gradient. The thermal and structural response of these members to fire is obtained via time-series integration by solving closedform equations at every time step. Emphasis is placed on the case of a wide-flanged perimeter column typical to North American construction, although this method could easily be applied to other member types (i.e. floor beams) or section shapes. The proposed methodology, referred to in this paper as the simplified member model, has three primary components: (1) a simplified thermal analysis, (2) a simplified prediction of structural capacity, and (3) a simplified prediction of structural demand. Together, components (2) and (3) comprise the simplified structural analysis shown in Fig. (1). The simplified member model can be used to analyze beam- 


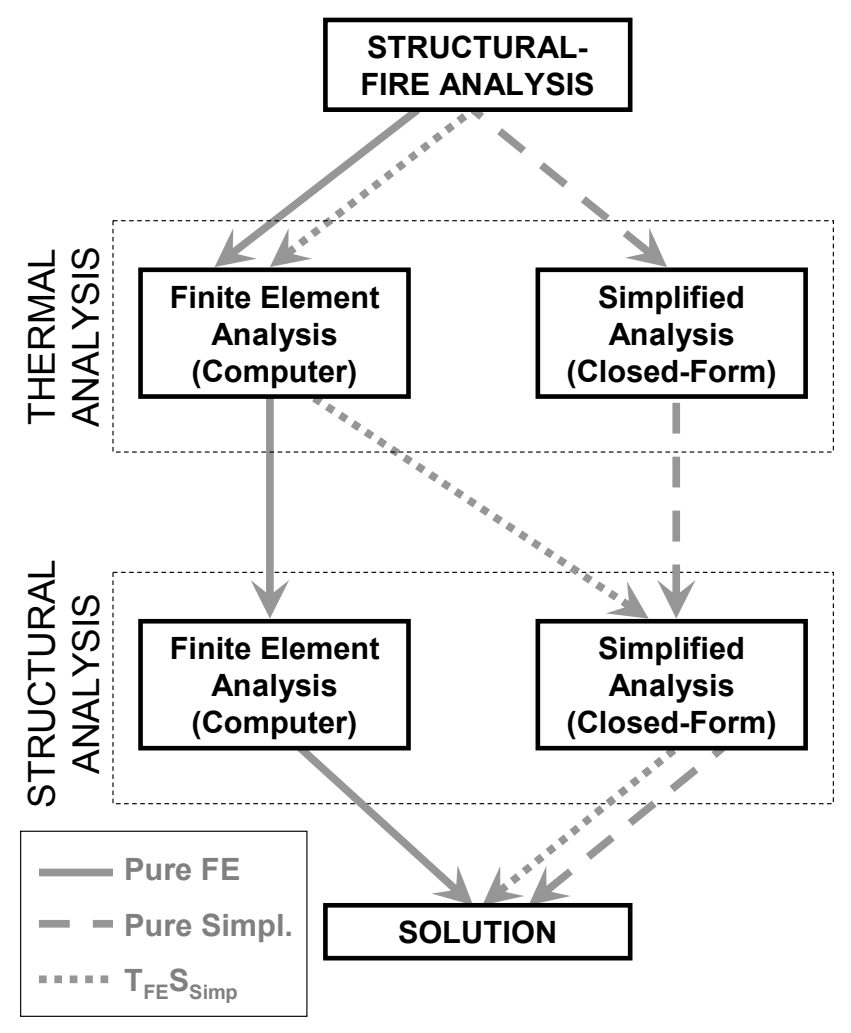

Fig. (1). Potential paths of a structural-fire analysis solution.

columns that develop thermal gradients in the direction of either the strong or weak axis. The simplified expressions presented in this paper can be solved in a spreadsheet or other closed-form mathematical algorithm because they require no iteration when solved at each time step, and these methods are sufficiently simple for use in a general performance-based design procedure for steel perimeter columns under fire.

Each component of the simplified member model is validated individually against computational results that have been discussed in previous studies [1,2]. Comparisons are then made between predictions of beam-column response based on computational results to that based on various combinations of the three simplified components. Fig. (1) shows three of the analysis combinations considered for this study: (1) a pure finite element solution (Pure FE) in which only computational tools are used, (2) a pure simplified solution (Pure Simplified) in which only simplified tools are used, and (3) a solution obtained via thermal FE analysis and simplified structural analysis $\left(\mathrm{T}_{\mathrm{FE}} \mathrm{S}_{\mathrm{Simp}}\right)$. It will be shown that each of the combinations considered provides a similar prediction of response and that the proposed simplified methods are sufficiently accurate to be used independently of computational analysis. The simplified methods have also been successfully validated with experimental studies discussed in [3].

\section{EXISTING METHODOLOGIES}

Reports such as that following the investigation of the fire-induced structural collapses at the World Trade Center by the National Institute of Standards and Technology (NIST) [4] have recommended the use of performance-based provisions to design structures to resist unwanted fire rather than prescriptive methods, which are the current state-ofpractice in the US. Performance-based methods examine the changes in demand and capacity that occur as a structural member is heated and consider the effects of its connection to the surrounding structure on its performance. Performance-based methods to calculate the capacity of heated steel members have been a part of the Eurocode for several years [5], and some performance-based provisions have also been included in the current edition of AISC's Steel Construction Manual [6]. Both references stipulate that an analysis of a steel structure's mechanical response must account for the degradation of steel material strength and stiffness due to increased temperatures as well as the effects of thermal expansions and large deflection. Simple methods are typically permitted only when the assumption of uniform temperature is reasonable while support and restraint conditions remain unchanged. Advanced calculation methods are recommended to capture the response of the structural frame to fire (particularly the interaction between connected structural members) and to consider the effects of non-uniform temperature. Both the Eurocode and AISC include guidelines for evaluating beam-column performance under fire, but both rely on the assumption of uniform section temperature.

Numerous studies have examined the relative accuracy of the simple analytical methods used by Eurocode and AISC to calculate the fire-exposed strength of steel beam-columns. For example, Takagi and Deierlein [7], Knobloch et al. [8], and Lopes et al. [9] have proposed adjustments to the codified strength provisions for steel beam-columns to improve their accuracy compared to the results of computational and experimental studies. However, all of these studies calculated beam-column strength under the assumption of uniform temperature.

To date, few simple analytical methods have been developed to calculate the response of fire-exposed steel beamcolumns, and even fewer have accounted for non-uniform temperature through the section depth. Most expressions, such as those proposed by Skowronski [10], Huang and Tan [11], and Li et al. [12], calculate the performance of a steel member as having either $P$ or $M$ (i.e. acting either a column or a beam) under the assumption of uniform temperature. One exception is a simple methodology proposed by Usmani et al. [13] which used compatibility-based solutions to calculate the response of fire-exposed members subjected to both $P$ and $M$. Simple calculations were made for sections with either a uniform or linearly varying temperature distribution through its depth. The results of the simple calculations compared well to corresponding results of computational modeling and fire tests. However, few other studies have proposed simple methods to calculate the mechanics of steel beam-columns that develop thermal gradients without resorting to an iterative or computational approach. The method proposed in this paper for simplified mechanical analysis of steel beam-columns with thermal gradients addresses this need.

A few simple methodologies have been proposed to calculate the thermal gradient that develops through the depth of a section whose fire exposure is not uniform. Most closedform approaches, such as those discussed by Gamble [14] and Buchanan [15] and included in the Eurocode [5], calcu- 


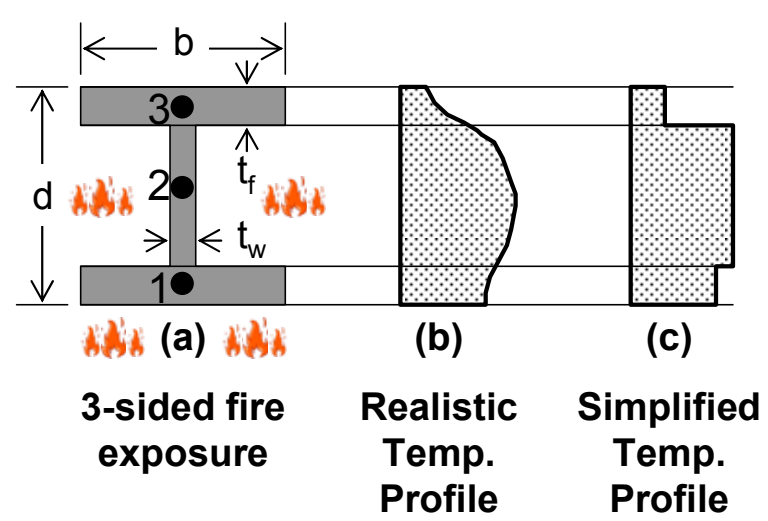

Fig. (2). Temperature profiles due to (a) 3-sided strong axis fire exposure: (b) realistic and (c) simplified.

late the temperature of fire-exposed steel members by considering the entire steel section to be a single lumped mass with which heat transfer with the surrounding environment is calculated. Ghojel and Wong [16] have proposed a lumped mass method with higher resolution, by which each plate of a wide-flanged floor beam (i.e. both flanges and the web) is approximated as a lumped mass. Their method explicitly calculates the temperature of each of these lumped masses and therefore can provide a simple, closed-form prediction of a non-uniform temperature profile. This method is only capable of predicting a gradient in the strong axis direction (i.e. parallel to the web) for members with no applied fire protection [16]. A more generalized variation of the method proposed by Ghojel and Wong is developed in this paper to calculate thermal gradients in both the strong and weak axis directions and account for the effects of applied fire protection. Few studies have proposed a methodology that uses the results of such a thermal analysis for a simplified calculation of mechanical behavior. It will be shown that the results of the proposed thermal methodology can easily be used as input for simplified structural analysis so that the total response of steel beam-columns to non-uniform fire exposure can be calculated as an explicit time-series integration.

\section{SIMPLIFIED THERMAL ANALYSIS}

A simplified, closed-form methodology for thermal analysis is proposed to calculate the temperature gradient that develops through the depth of a steel cross-section due to non-uniform heating. The analysis is based on the simplified methodology proposed by Ghojel and Wong [16] by which the beam-column cross-section is discretized into a small number of coarse fibers. Heat transfer between these multiple lumped masses and the surrounding environment is then calculated as a time-series integration via closed-form expressions to obtain the temperature distribution through the cross-section.

\section{Coarse-Fiber Temperature Profile}

Fig. (2(a)) shows a section that develops a gradient in the direction of the strong axis (and therefore bends about this axis) broken down into three fibers, one for each flange and for the web. The weak axis section (Fig. 3(a)) is divided into four total fibers: three fibers of equal size along the width of the flange and one for the web. Note that only one flange in

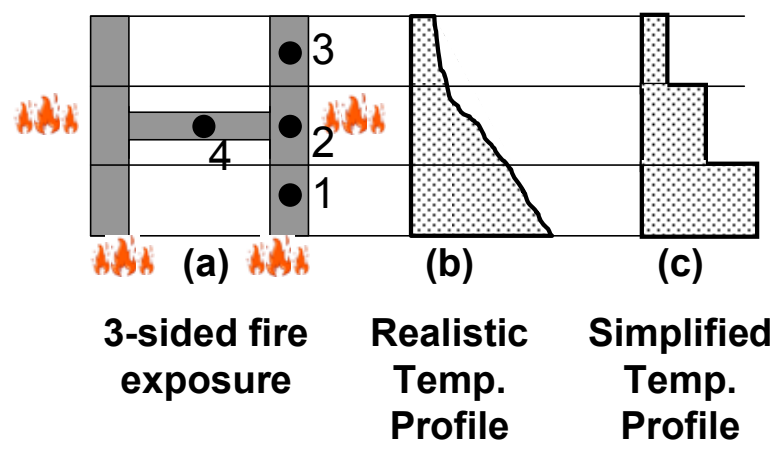

Fig. (3). Temperature profiles due to (a) 3-sided weak axis fire exposure: (b) realistic and (c) simplified.

the weak axis cross-section is modeled due to symmetry. When analysis is performed, the area of the flange fibers is doubled to account for the response of the entire section. For the rest of this paper, the area of each weak axis flange fiber $j\left(A_{s, j}\right)$ will equal 2(b/3)t $t_{f}$ (where $b$ and $t_{f}$ are defined in Fig. $(\mathbf{2}(\mathbf{a}))$, twice the area of each individual flange fiber shown in Fig. (3(a)).

Figs. (2(b)) and (3(b)) show realistic representative temperature distributions that develop in steel wide-flanged sections in the direction of the strong and weak axis, respectively, due to the three-sided heating orientations shown in Figs. (2(a) and 3(a)). Currently available computational analysis tools are capable of obtaining these thermal profiles via FE analysis of cross-sections modeled with high levels of discretization. However, these non-uniform temperature distributions are not conducive to simple analysis, and therefore they are simplified for the proposed methodology as shown in Figs. (2(c) and 3(c)). The temperature in each fiber in Figs. (2(a) and 3(a)) is assumed to be constant and equal to the average of the temperatures across that fiber in the realistic profile. This transformation allows a relatively straightforward calculation of the temperature effects in each coarse fiber. The simplified thermal profile can be calculated either from realistic thermal profiles (obtained from computational FE modeling of highly discretized cross-sections or from fire tests) or from simplified heat transfer analysis (by considering the fibers as individual lumped masses).

\section{Multiple Lumped Mass Analysis}

Each of the coarse fibers in the sections shown in Figs. (2(a) and 3(a)) are approximated as a discrete lumped mass to which heat transfer with the surrounding environment and with adjacent fibers can be calculated. Each lumped mass is assumed to have a uniform temperature, which can be used to construct the coarse-fiber temperature profiles shown in Figs. (2(c) and 3(c)). For a cross-section that develops a thermal gradient in the strong axis direction due to three-sided fire exposure, the coarse fibers (Fig. 4(a)) are transformed into three lumped masses, one for each flange and one for the web, located at their respective centers of gravity (Fig. 4(b)). Fig. (4(b)) shows the heat transfer from the hot gases to each lumped mass at its fire-exposed surfaces $\left(Q_{i n}\right)$, from each lumped mass to the ambient environment at its unexposed surfaces $\left(Q_{\text {out }}\right)$, and between lumped masses (for example $Q_{1-2}$ ). Applying the law of the conservation of energy yields the following equations that can be used to calcu- 


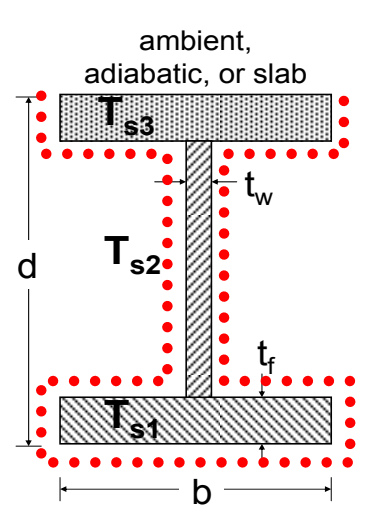

(a)

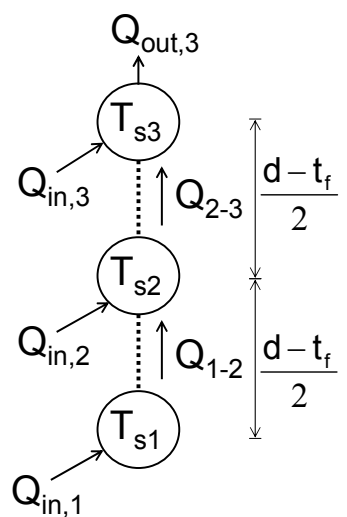

(b)

Fig. (4). Approximation of the (a) strong axis cross-section with 3sided fire exposure as (b) an equivalent system of lumped masses.

late the change in temperature between time steps $i$ and $i-1$ $\left(\Delta T_{s}=T_{s(i)}-T_{s(i-1)}\right)$ for each lumped mass in the strong axis section:

$$
\begin{aligned}
& \frac{\Delta T_{s, 1}}{\Delta t}\left(\rho_{s} c_{s, 1} A_{s, 1}+\frac{\rho_{p} c_{p, 1} d_{p} P_{p, 1}}{2}\right)=Q_{i n, 1}-Q_{1-2} \\
& \frac{\Delta T_{s, 2}}{\Delta t}\left(\rho_{s} c_{s, 2} A_{s, 2}+\frac{\rho_{p} c_{p, 2} d_{p} P_{p, 2}}{2}\right)=Q_{i n, 2}+Q_{1-2}-Q_{2-3} \\
& \frac{\Delta T_{s, 3}}{\Delta t}\left(\rho_{s} c_{s, 3} A_{s, 3}+\frac{\rho_{p} c_{p, 3} d_{p} P_{p, 3}}{2}\right)=Q_{i n, 3}+Q_{2-3}-Q_{\text {out }, 3}
\end{aligned}
$$

where $\rho_{s}$ and $c_{s}$ are the density $\left(\mathrm{kg} / \mathrm{m}^{3}\right)$ and specific heat $(\mathrm{J} / \mathrm{kg}-\mathrm{K})$ of steel; $\rho_{p}, c_{p}$, and $d_{p}$ are the density, specific heat, and thickness (m) of the fire protection; $A_{s}$ is the crosssectional area of the steel fiber $\left(\mathrm{m}^{2}\right) ; P_{p}$ is the fire exposed perimeter $(\mathrm{m})$ of the fire protection; and $\Delta t$ is the time step (sec). A general derivation of the components of Eq. 1 is provided by Ghojel and Wong [16].

Likewise, the coarse fibers in the weak axis section (Fig. 5(a)) are transformed into a system of four lumped masses, one for the web and one each for the three equal divisions along the width of the flange (Fig. 5(b)), from which a set of heat transfer equations similar to Eq. 1 can be derived:

$$
\begin{aligned}
& \frac{\Delta T_{s, 1}}{\Delta t}\left(\rho_{s} c_{s, 1} A_{s, 1}+\frac{\rho_{p} c_{p, 1} d_{p} P_{p, 1}}{2}\right)=Q_{i n, 1}-Q_{1-2} \\
& \frac{\Delta T_{s, 2}}{\Delta t}\left(\rho_{s} c_{s, 2} A_{s, 2}+\frac{\rho_{p} c_{p, 2} d_{p} P_{p, 2}}{2}\right)=Q_{i n, 2}+Q_{1-2}-Q_{2-3}-Q_{2-4}-Q_{o u t, 2} \\
& \frac{\Delta T_{s, 3}}{\Delta t}\left(\rho_{s} c_{s, 3} A_{s, 3}+\frac{\rho_{p} c_{p, 3} d_{p} P_{p, 3}}{2}\right)=Q_{\text {in }, 3}+Q_{2-3}-Q_{\text {out }, 3} \\
& \frac{\Delta T_{s, 4}}{\Delta t}\left(\rho_{s} c_{s, 4} A_{s, 4}+\frac{\rho_{p} c_{p, 4} d_{p} P_{p, 4}}{2}\right)=Q_{i n, 4}+2 Q_{2-4}-Q_{\text {out }, 4}
\end{aligned}
$$

The first term in parentheses in Eqs. 1 and 2 represents the heat energy absorbed by the lumped mass' steel during the time step. The second term represents the heat absorbed by the fire protection applied to the lumped mass. If no fire protection is applied, then this term reduces to zero. Eqs. 1 and 2 can be easily rearranged to solve for the steel temperature of each lumped mass at the current time step $\left(T_{s(i)}\right)$.

Different formulations for $Q_{\text {in }}$ and $Q_{\text {out }}$ are used depending on whether the surfaces for which they are calculated are unprotected or are coated with a layer of fire protection with thickness $d_{p}$ :

Unprotected:

$$
\begin{aligned}
& Q_{\text {in }, j}=P_{u, i n, j}\left[h\left(T_{\text {fire }}-T_{s, j(i-1)}\right)+\sigma \varepsilon\left(\left(T_{\text {fire }}+273\right)^{4}-\left(T_{s, j(i-1)}+273\right)^{4}\right)\right] \\
& Q_{\text {out }, j}=P_{u, \text { out }, j}\left[h\left(T_{s, j(i-1)}-T_{\text {ambient }}\right)+\sigma \varepsilon\left(\left(T_{s, j(i-1)}+273\right)^{4}\right.\right. \\
& \left.\left.-\left(T_{\text {ambient }}+273\right)^{4}\right)\right]
\end{aligned}
$$

Protected:

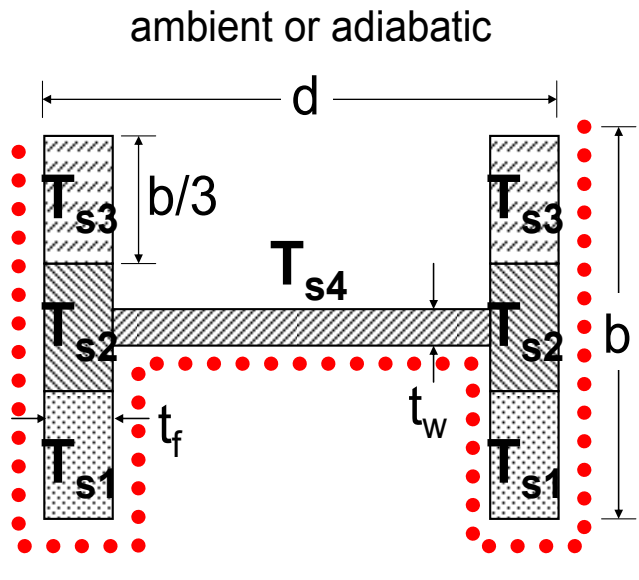

(a)

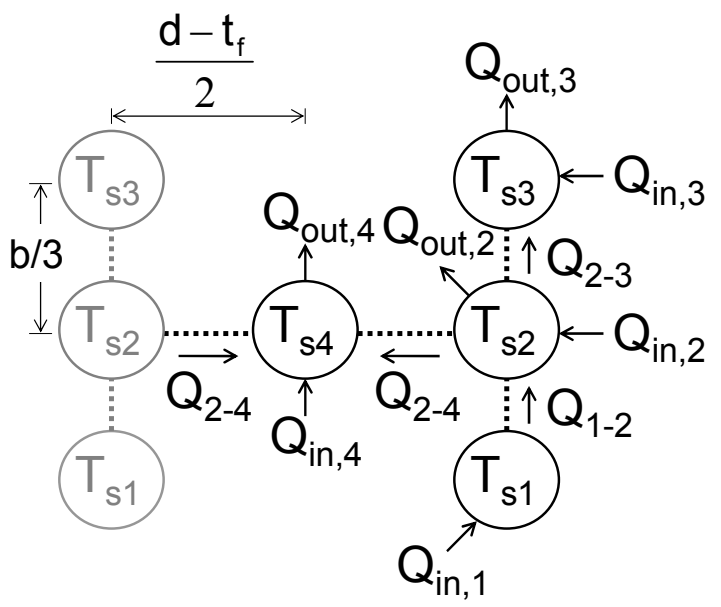

(b)

Fig. (5). Approximation of the (a) weak axis cross-section with 3-sided fire exposure as (b) an equivalent system of lumped masses. 
$Q_{i n, j}=\frac{P_{p, i n, j} k_{p, j}\left(T_{\text {fire }}-T_{s, j(i-1)}\right)}{d_{p}}$

$Q_{\text {out }, j}=\frac{P_{p, \text { out }, j} k_{p, j}\left(T_{s, j(i-1)}-T_{\text {ambient }}\right)}{d_{p}}$

where $j$ is the fiber number; $i$ denotes the time step; $T_{\text {fire }}$ is the fire temperature $\left({ }^{\circ} \mathrm{C}\right) ; T_{s(i-1)}$ is the temperature of the steel $\left({ }^{\circ} \mathrm{C}\right)$ at the previous time step $i-1 ; k_{s}$ and $k_{p}$ are the thermal conductivity $(\mathrm{W} / \mathrm{m}-\mathrm{K})$ of the steel and fire protection, respectively; $P_{u}$ is the perimeter $(\mathrm{m})$ of the unprotected steel surface; $h$ is the convective heat transfer coefficient $\left(\mathrm{W} / \mathrm{m}^{2}\right.$ $\mathrm{K}) ; \varepsilon$ is the relative emissivity; and $\sigma$ is the StefanBoltzmann constant $\left(56.7 * 10^{-9} \mathrm{~W} / \mathrm{m}^{2}-\mathrm{K}^{4}\right) . T_{\text {fire }}$ is taken as the fire temperature halfway through the current time step $i$ (i.e. at time $\left.t_{(i-1)}+\Delta t / 2\right)$ as an approximation of the fire conditions through the length of the time step [14]. The in or out subscripts attached to $P$ denote the perimeter that is exposed or unexposed to fire, respectively.

Heat transfer equations for unprotected surfaces (Eq. 3) include both convective and radiative heat transfer. However, these coefficients are not included in the heat transfer equations for protected surfaces. For Eq. 4, it is assumed that the external surface of the insulation has the same temperature as the fire and the internal surface has the same temperature as the steel. The gradient through the thickness of the fire protection between these temperatures is conservatively assumed to be linear, and heat transfer through the insulation is calculated using this gradient in Eq. 4 [15]. The second term in parentheses in Eqs. 1 and 2 also reflects this linear gradient assumption by including a factor of 2 in the denominator (i.e. the temperature of the fire protection equals the average of the fire and steel temperatures). Eurocode replaces the 2 in the denominator of this term with 3 to reduce the conservatism of the linear thermal gradient assumption [5].

Heat transfer between lumped masses $a$ and $b\left(Q_{a-b}\right)$ assumes that the linkage between them has a linear thermal gradient:

$Q_{a-b}=\frac{t_{a-b}\left(T_{s, a(i-1)}-T_{s, b(i-1)}\right)}{y_{a-b}}\left(\frac{k_{s, a}+k_{s, b}}{2}\right)$

where $y_{a-b}$ is the distance between the centers of gravity of the lumped masses, and $t_{a-b}$ is the thickness of the steel plate that serves as a thermal pathway between them. The value of $t_{a-b}$ is assumed to be $t_{w}$ for $Q_{a-b}$ between adjacent flange and web lumped masses (e.g. $Q_{1-2}$ and $Q_{2-3}$ in Fig. $(\mathbf{4}(\mathbf{b}))$ and $Q_{2-4}$ in Fig. (5(b)) and $t_{f}$ for $Q_{a-b}$ between adjacent flange lumped masses (e.g. $Q_{1-2}$ and $Q_{2-3}$ in Fig. 5(b)). The thermal conductivity between lumped masses is approximated as the average between the thermal conductivity of each, shown as the second term in Eq. 5.

Note that the thermal conductivity $(k)$ and specific heat (c) of both the steel and fire protection in Eqs. 1 through 5 include a subscript corresponding to their lumped mass. Realistically, these material properties are functions of temperature, and they are modeled as such in this methodology. Computational FE solutions use iterative procedures at every time step to calculate the temperature of each fiber. Each fiber's temperature-dependent thermal properties are continuously updated until convergence is achieved and these properties correspond to the fiber's temperature at that time step. To avoid iteration, temperature-dependent thermal properties for steel at the current time step are calculated for each lumped mass according to its steel temperature at the previous time step $i-l\left(T_{s(i-1)}\right)$. To calculate updated material properties for the fire protection, the temperature of the fire protection is approximated as the average of $T_{\text {fire }}$ at the current time step (as before, calculated for time $t_{(i-1)}+\Delta t / 2$ ) and $T_{s(i-1)}$. This "one-step lag" strategy was successfully used by Gamble [14] to update the temperature-dependent properties of steel and fire protection during analysis of whole crosssections via a single lumped mass. It will be shown that updating thermal properties according to temperatures from the previous time step provides a sufficiently accurate calculation of steel temperature in each lumped mass fiber as long as the time step remains sufficiently short (i.e. no more than one minute).

\section{SIMPLIFIED PREDICTION OF CAPACITY}

A simplified methodology is proposed to predict the plastic capacity of steel beam-columns that develop a thermal gradient through their depth due to non-uniform fire exposure, as in the case of a perimeter column, using the coarsefiber cross-sectional model described previously. The proposed methodology can be used for beam-columns that develop gradients in the direction of either the strong or weak axis (which thereby induce bending in the same direction). Though not discussed in this paper, buckling must also be considered when calculating the total response of beamcolumns to fire. Simple design equations to calculate local buckling strength via stress-based $[3,17]$ or strain-based methods [18], lateral-torsional buckling strength [19], or flexural buckling strength [8] of beam-columns could be added to this procedure to include additional limit states.

\section{Calculating Plastic Capacity}

The plastic capacity of a steel cross-section to resist combinations of $P$ and $M$ is calculated using the same procedure proposed by the authors [1], except that this methodology uses the much coarser cross-sectional discretizations shown in Figs. (2(a) and 3(a)). Temperatures for the lumped masses in this coarse discretization are obtained using the simplified thermal methodology discussed above. Fig. (6(b)) shows the simplified thermal profile that develops due to three-sided heating of the section shown in Fig. $(\mathbf{6}(\mathbf{a}))$. The yield strength for each fiber, $j$, is calculated as a function of its temperature. Assuming that the temperature in each fiber has exceeded the value corresponding to a reduction in yield stress, the $\sigma_{y}$ profile corresponding to the simplified thermal profile in Fig. $(\mathbf{6}(\mathbf{b}))$ is represented by Fig. $(\mathbf{6}(\mathbf{c}))$ - the larger the temperature, the smaller the $\sigma_{y}$ of the steel material. The capacity of the fully yielded section, $P_{y}$, is calculated by integrating $\sigma_{y}$ of each fiber times its area through the depth of the section, where $\sigma_{y}$ has the same sign for all fibers (i.e. all in compression or all in tension as shown in Figs. $(\mathbf{6}(\mathbf{c}))$ and (g)). The integration for $P$ shown in [1] can be approximated as a summation over all fibers (i.e. 3 fibers for a strong axis section and 4 fibers for a weak axis section):

$$
P=\int_{A} \sigma_{y} d A=\sum_{j} \sigma_{y, j} A_{j}
$$




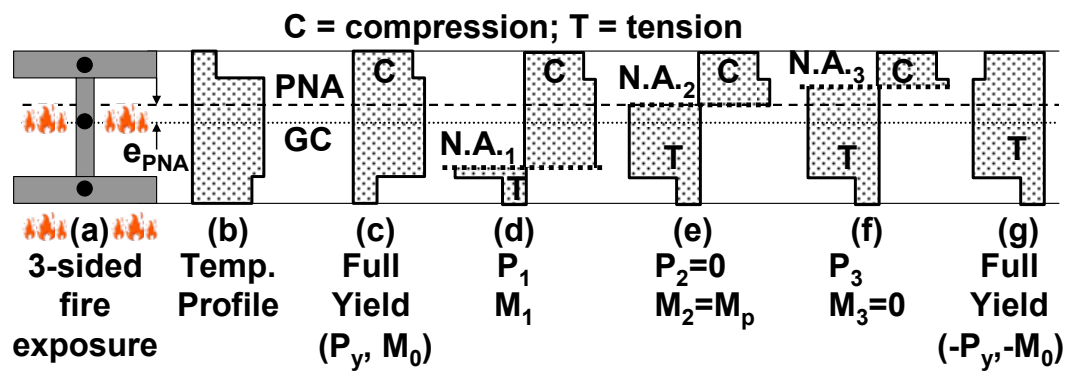

Fig. (6). Temperature and yield stress profiles with the position of the neutral axial (NA) at various locations.

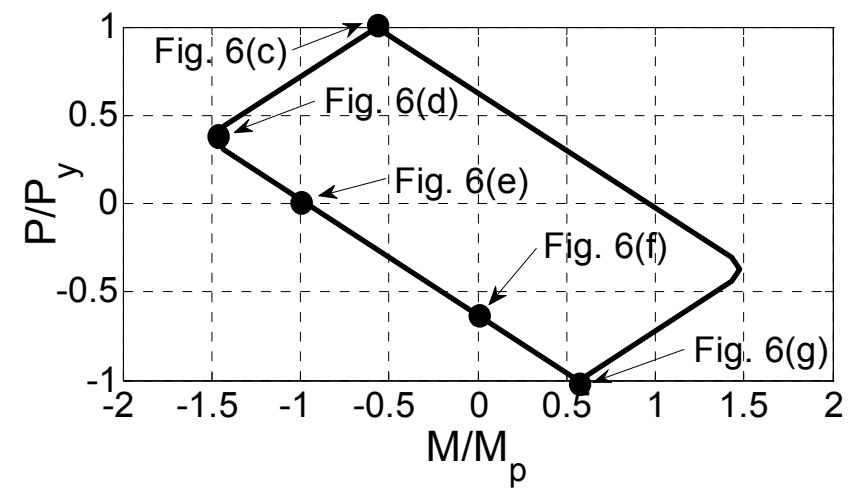

Fig. (7). Representative plastic $P-M$ capacity envelope for a strong axis cross-section with a thermal gradient.

Likewise, the integration for moment is approximated with a summation of $\sigma_{y, j}$ times the distance $\left(y_{j}\right)$ to the reference axis (chosen to be the section's geometric centroid) times $A_{j}$, where the sign of $\sigma_{y, j}$ depends on its relative position to the neutral axis (NA):

$$
M=\int_{A} \sigma_{y} y d A=\sum_{j} \sigma_{y, j} y_{j} A_{j}
$$

The NA represents the axis of zero strain, and the plastic neutral axis (PNA) represents the NA position when the resultant of the compressive and tensile stresses in a fully yielded section are equal (i.e. $\mathrm{C}=\mathrm{T}$ as shown in Fig. $\mathbf{6}(\mathbf{e})$ ). The location of the PNA is therefore determined by finding the NA position at which the total axial load $P$ (Eq. 6) is zero. The plastic moment capacity of the section $\left(M_{p}\right)$ can then be calculated via Eq. 7 about the geometric centroid based on the PNA stress distribution shown in Fig. (6(e)).

As discussed in [1], each point on the plastic $P-M$ capacity envelope represents a specific location of the NA. Therefore, the plastic $P-M$ capacity envelope at every time step is calculated by incrementally moving the NA through the depth of the section and integrating the stresses according to Eqs. 6 and 7 (which are functions solely of temperature and thus are closed-form) at each NA location. Fig. (7) shows a representative plastic $P-M$ capacity envelope obtained using the simplified summation calculations, in which the values of $P$ and $M$ have been normalized by $P_{y}$ and $M_{p}$, respectively. Fig. (7) also shows the positions of the five discrete points on the envelope that are represented by Figs. $(\mathbf{6}(\mathbf{c}))$ through $(\mathrm{g})$.

In the proposed simplified methodology, it is recommended that Eqs. 6 and 7 be solved at a minimum of ten NA locations (i.e. at ten evenly spaced increments through the depth of the section) in order to obtain an adequate number of points to accurately construct the plastic $P-M$ capacity envelope. Also, using at least ten NA locations ensures that one of them will be sufficiently close to the PNA, providing an accurate calculation of $M_{p}$. As will be shown later in this paper, using fewer NA locations may predict unconservative values of $M_{p}$ once a significant gradient emerges through the depth of the section.

\section{SIMPLIFIED PREDICTION OF DEMAND}

A simplified method to predict the demand on steel beam-columns that develop thermal gradients is developed for the case of the perimeter column whose cross-section is modeled with the coarse-fiber temperature profile described previously. The methods described in this section could also be used to calculate the demand of other member types that develop thru-depth thermal gradients and act as beamcolumns when heated, such as floor beams. A material model for structural steel that approximates non-linearity and considers temperature effects is used as a simplified alternative to the Eurocode material model for steel. The following subsections outline the construction of the simplified material model as well as procedures to calculate the axial load and moment experienced by the beam-column due to the thermal gradient. The last subsection describes the overall step-by-step procedure for the simplified calculation of demand.

The idealized structural model shown in Fig. $(\mathbf{8}(\mathbf{a}))$ is used to approximate the loading and boundary conditions of a one-story length of a perimeter column. The sign convention for this study is such that positive moments produce tension in the left-hand (cooler) face of the member, and positive axial forces correspond to compression. The member is assumed to be restrained from rotating at its top and bottom due to its connection to adjacent members and will therefore develop constant moment along its length when a thru-depth thermal gradient develops (Fig. 8(a)). Axial load $P$ represents the applied load, and its value may increase because axial thermal expansion is opposed by a vertical spring whose stiffness, $k_{\text {spring }}$, represents that provided by the adjacent structure. The spring is assumed to only provide resistance against upward displacement from the column's initially loaded position in order to conservatively prevent the column from "hanging" from the spring (thus alleviating its axial load) once it significantly weakens and downward displacements become large.

As discussed in [2], two moment reactions will develop in response to the thermal gradient. "Thermal bowing" mo- 


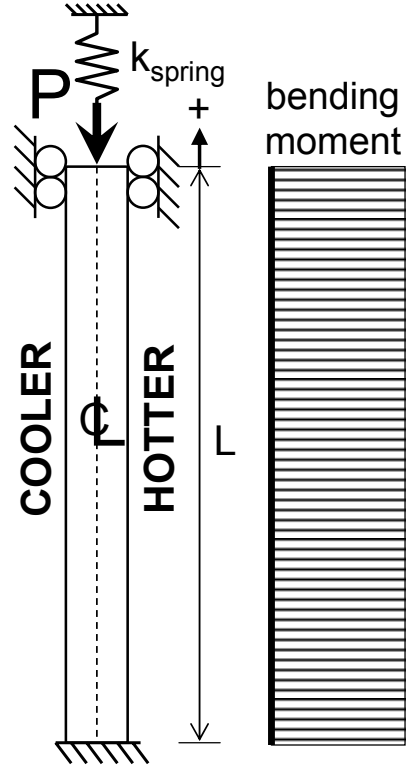

(a)

(b)

Fig. (8). Loading and boundary condition used to approximate (a) a perimeter column, shown with (b) bending moment diagram.

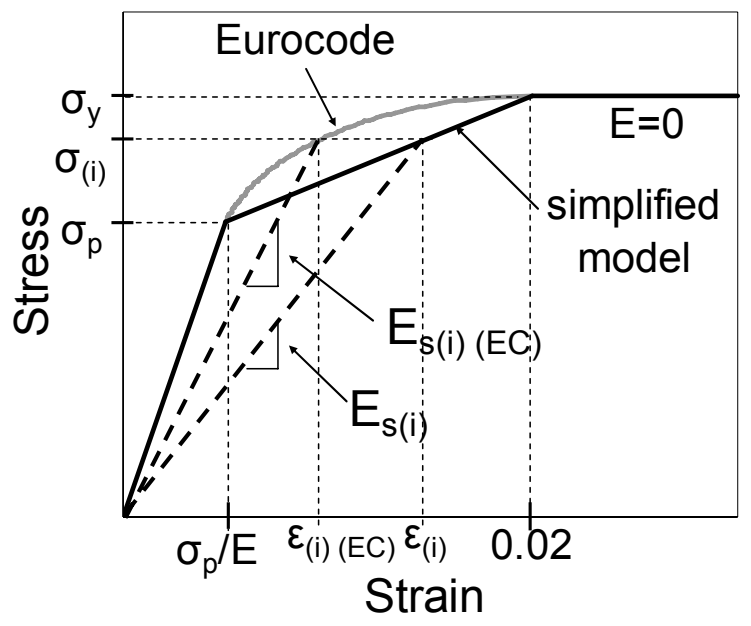

Fig. (9). Material models for structural steel: Eurocode compared to a simplified tri-linear model.

ment, $M_{T}$, initially develops in response to the temperature variation, and thus the strain variation, between the cooler and hotter regions of the cross-section. Since the right face is hotter than the left face, the right face will undergo a larger thermal expansion. Since the ends are restrained from rotating, the hotter face becomes compressed and the cooler face experiences tension, leading to a positive moment according to the sign convention. The second moment develops when the temperatures become high enough to reduce the modulus of elasticity $(E)$, which is not uniform through the section's depth due to the thermal gradient. At this point, the section's center of stiffness (i.e. the effective centroid) migrates toward the cooler face of the cross section [2]. An axial load applied at the geometric centroid, which now does not coincide with the effective centroid, generates a negative mo- ment, $M_{P^{*} e}$, equal to $P$ times the distance between the effective and geometric centroids.

Second order member $(P-\delta)$ effects are assumed to be negligible. In addition, second order frame $(P-\Delta)$ effects are not considered because the columns studied here do not deflect laterally at their supports. Although the interaction of the perimeter column and the floor system may have some effect on the performance of perimeter columns with temperatures above $400^{\circ} \mathrm{C}$ [20], the approximation of constant moment along the column length in this case is reasonable and conservative [3].

\section{Simplified Steel Material Model}

The steel material model used for this methodology is a simplification of that specified by Eurocode [5], which uses temperature-based reduction factors for steel strength and stiffness. The yield stress $\left(\sigma_{y}\right)$ is equal to the yield stress at ambient temperature $\left(\sigma_{y, 20}\right)$ multiplied by $k_{y, T}$, a yield stress reduction factor that becomes less than 1.0 when temperatures exceed $400^{\circ} \mathrm{C}$. Similarly, the reduction factor, $k_{E, T}$, for the modulus of elasticity $(E)$ becomes less than 1.0 when temperatures exceed $100^{\circ} \mathrm{C}$. The stress-strain relationship, which is elastic perfectly plastic at room temperature, is transformed into a non-linear relationship at elevated temperatures (i.e. the proportional limit stress, $\sigma_{p}$, no longer equals the yield stress as shown in Fig. 9). This nonlinearity begins when temperatures exceed $100^{\circ} \mathrm{C}$ as represented by $k_{p, T}$, which equals the proportional limit stress divided by the yield stress at ambient temperature [5]. Fig. (9) compares the non-linear Eurocode stress-strain model for structural steel to that of the simplified material model. The stress-strain relationship is approximated as a tri-linear function with a constant tangent modulus $\left(E_{t}\right)$ in the stress region between $\sigma_{p}$ and $\sigma_{y}$ (shown as the "simplified model" in Fig. 9). The strain at yield is chosen to be 0.02 as recommended by Eurocode [5]. The linear approximation in the stress region between $\sigma_{p}$ and $\sigma_{y}$ is used to simplify the stress-strain calculations that are made when plastic strain develops.

Fig. (10) shows that when the material is loaded beyond its proportional limit into the tangent region at a given temperature during time step $i\left(T_{(i)}\right)$, plastic strain develops in the steel material. The plastic strain accumulated during this step, $\varepsilon_{p l(i)}$, can be calculated from the geometry of the trilinear material model based on the position of $\sigma_{(i)}$, represent-

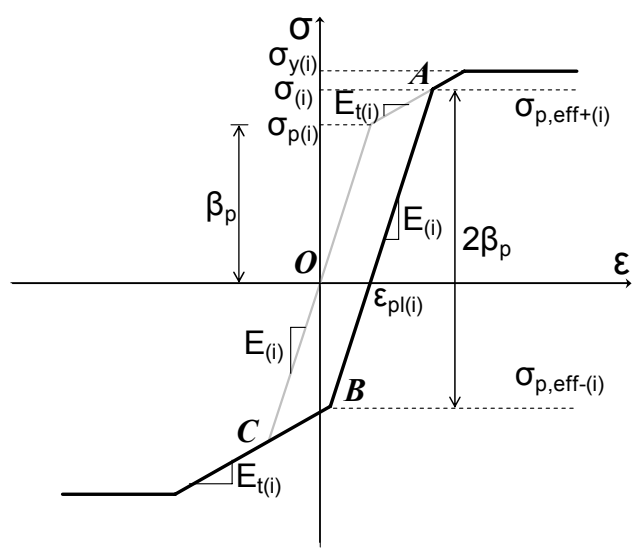

Fig. (10). Simplified material model with plastic strain. 
ing the stress at time step $i$, between $\sigma_{p(i)}$ and $\sigma_{y(i)}$. The plastic strain, rather than the maximum stress level, describes the complete stress-strain history as the steel temperature changes [21]. As the material unloads from point $\boldsymbol{A}$, it travels the path $\boldsymbol{A B C}$, which has a non-zero value of $\varepsilon_{p l(i)}$ - the material no longer passes through the origin at point $\boldsymbol{O}$. The simplified model unloads with elastic modulus $E_{(i)}$ over a stress range of $2 \beta_{p}$ (where $\beta_{p}$ is equal to $\sigma_{p(i)}$ ) regardless of the value of $\varepsilon_{p l(i)}$ [21], and the portion of load path $\boldsymbol{A B C}$ for negative stresses rejoins the original material model via the tangent stiffness $E_{t(i)}$. Calculations using the Eurocode model when plastic strains have developed are more complicated; for example, the construction of negative-stress portion of pathway $\boldsymbol{A} \boldsymbol{B C}$ requires the use of several non-linear branches to rejoin the original material model $\boldsymbol{A O C}$ [21]. The simplified model is therefore used as a simpler alternative.

Note that the tri-linear model will estimate a larger $\varepsilon_{p l(i)}$ for a given value of $\sigma_{(i)}$ than the Eurocode model. Likewise, strains at each time step, $\varepsilon_{(i)}$, for a given value of $\sigma_{(i)}$ between $\sigma_{p(i)}$ and $\sigma_{y(i)}$ will also be larger when calculated with the simplified model (see Fig. 9). The methodology proposed in this paper applies an adjustment factor to account for this error. The magnitude of this adjustment factor will be discussed later in this paper, and the effectiveness of using the simplified material model in this fashion will be confirmed via comparison to computational results. Experimental validation of this adjustment factor is also provided in [3].

The total strain of every fiber in the beam-column crosssection $\left(\varepsilon_{\text {total }}\right)$ is comprised of the mechanical strain $\left(\varepsilon_{\sigma, j}\right)$ plus the thermal strain $\left(\varepsilon_{T, j}\right)$, which equals the coefficient of thermal expansion times the change in the fiber's temperature $\left(\alpha \Delta T_{j}\right)$. Note that $\varepsilon_{\text {total }}$ does not have a subscript $j$ representing the fiber. For the column shown in Fig. $(\mathbf{8}(\mathbf{a}))$, the rotation of each end is restrained, and therefore the curvature is zero and $\varepsilon_{\text {total }}$ will be constant through the section depth since all fibers translate uniformly in the vertical direction. To calculate the $P-M$ response of the coarsely discretized crosssection, the secant modulus, $E_{s, j}$, is used as a generalized description of each fiber's stiffness [22,23], as shown in Fig. (9) for time step $i$ :

$$
E_{s, j}=\frac{\sigma_{j}}{\varepsilon_{\sigma, j}}
$$

When $\sigma_{j}<\sigma_{p, j}, E_{s, j}$ equals the modulus of elasticity, reduced as a function of temperature $\left(E_{j}=E_{20} k_{E, T, j}\right)$. Once $\sigma_{j}>$ $\sigma_{p, j}, E_{s, j}$ does not represent the true stiffness of each fiber but rather a relative measure of stiffness between fibers which will be shown to be sufficient for predicting structural response. Fig. (9) shows that the simplified steel material model underestimates $E_{s}$ compared to the Eurocode model. As for the overestimation of strain, this error will be accounted for via an adjustment factor when demand is calculated.

Note that $E_{s, j}$ is a function of both the temperature at time step $i$ and the corresponding stress-strain state of fiber $j$. Computational FE analysis tools use iterative methods that update the value of $E_{s, j}$ at every iteration so that it corresponds directly to the column's temperature and stress-strain state at that time step. To avoid iteration, the proposed methodology calculates the values for $E_{s, j}$ at time step $i$ using the stress and strain from time step $i$ - 1 , similar to the "one-step lag" strategy used to update temperature-dependent material properties in the simplified thermal analysis outlined in Section 3 [14]. It will be shown later in this paper that this simplification provides reasonably accurate results.

\section{Calculating $\boldsymbol{P}$}

The magnitude of axial load $P$ at time step $i$ is calculated with the following expression, which accounts for the resistance of the vertical spring in Fig. $(\mathbf{8}(\mathbf{a}))$ to vertical thermal expansion:

$$
P=P_{o}+\left(\varepsilon_{\text {total }}-\varepsilon_{o}\right) k_{\text {spring }} L \geq P_{o}
$$

$P_{o}$ and $\varepsilon_{o}$ represent the initial applied axial load and initial strain experienced by the column, and $L$ is the length of the column as shown in Fig. $(\mathbf{8}(\mathbf{a}))$. Recall that $\varepsilon_{\text {total }}$ equals the sum of $\varepsilon_{T, j}$ and $\varepsilon_{\sigma, j}-$ its value describes the total axial elongation of the column in response to heating and is the same for every fiber at each time step. The total strain is adjusted by $\varepsilon_{o}$ so that only upward displacements from the initially loaded position can induce an increase in $P$. For the same reason, the minimum magnitude of $P$ in Eq. 9 is restricted to $P_{o}$.

\section{Calculating $M_{T}$}

To calculate $M_{T}$ at time step $i$, the analyst needs to determine the mechanical (or stress-related) strains induced via resistance to thermal curvature at every fiber $j\left(\varepsilon_{\sigma, T, j}\right)$. Mechanical strain $\varepsilon_{\sigma, j}$ is comprised of $\varepsilon_{\sigma, T, j}$ plus $\varepsilon_{\sigma, P, j}$, which is the mechanical strain induced by applied load $P$. The authors have previously shown that $M_{T}$ can be calculated based on $\varepsilon_{T, j}, E_{s, j}, A_{j}$, and $y_{j}$ only [2]. As mentioned previously, $E_{s, j}$ is calculated according to $\sigma_{j}$ and $\varepsilon_{\sigma, j}$ from time step $i$ - 1 . Assuming that all fibers are in equilibrium and that the column cross-section remains horizontal (i.e. $\varepsilon_{\text {total }}$ is constant for all fibers), $M_{T}$ is calculated with the following expression:

$$
M_{T}=\eta \sum_{j} \varepsilon_{\sigma, T, j} E_{s, j} y_{j} A_{j}=\eta \sum_{j}\left(\frac{\sum \varepsilon_{T, j} E_{s, j} A_{j}}{\sum E_{s, j} A_{j}}-\varepsilon_{T, j}\right) E_{s, j} y_{j} A_{j}
$$

Adjustment factor $\eta$ is included in Eq. 10 to address the overestimation of strain (and subsequent underestimation of $E_{s, j}$ ) that occurs when the simplified trilinear material model for steel is used instead of the Eurocode model (see Fig. 9). In the course of this study, the authors have found that using a value of $\eta=0.75$ produces good agreement with analysis results of computational models that account for realistic material, geometric, and thermal non-linearities as will be discussed later in this paper.

\section{Calculating $M_{P *}$}

To calculate $M_{P^{*} e}$ at time step $i$, the position of the effective centroid $\left(y_{E C}\right)$ must be calculated. The effective centroid represents the center of stiffness in a steel cross-section - it is the location through which the axial load must be applied to produce pure axial stress with no bending. The effective centroid of a cross-section with a uniform temperature profile will coincide with its geometric centroid. Assuming that the maximum temperature is high enough to cause a reduction in stiffness (i.e. $E_{s, j}$ ), $y_{E C}$ for a cross-section with a nonuniform temperature profile will migrate toward to cooler parts of the cross-section with higher stiffness. Using a simi- 


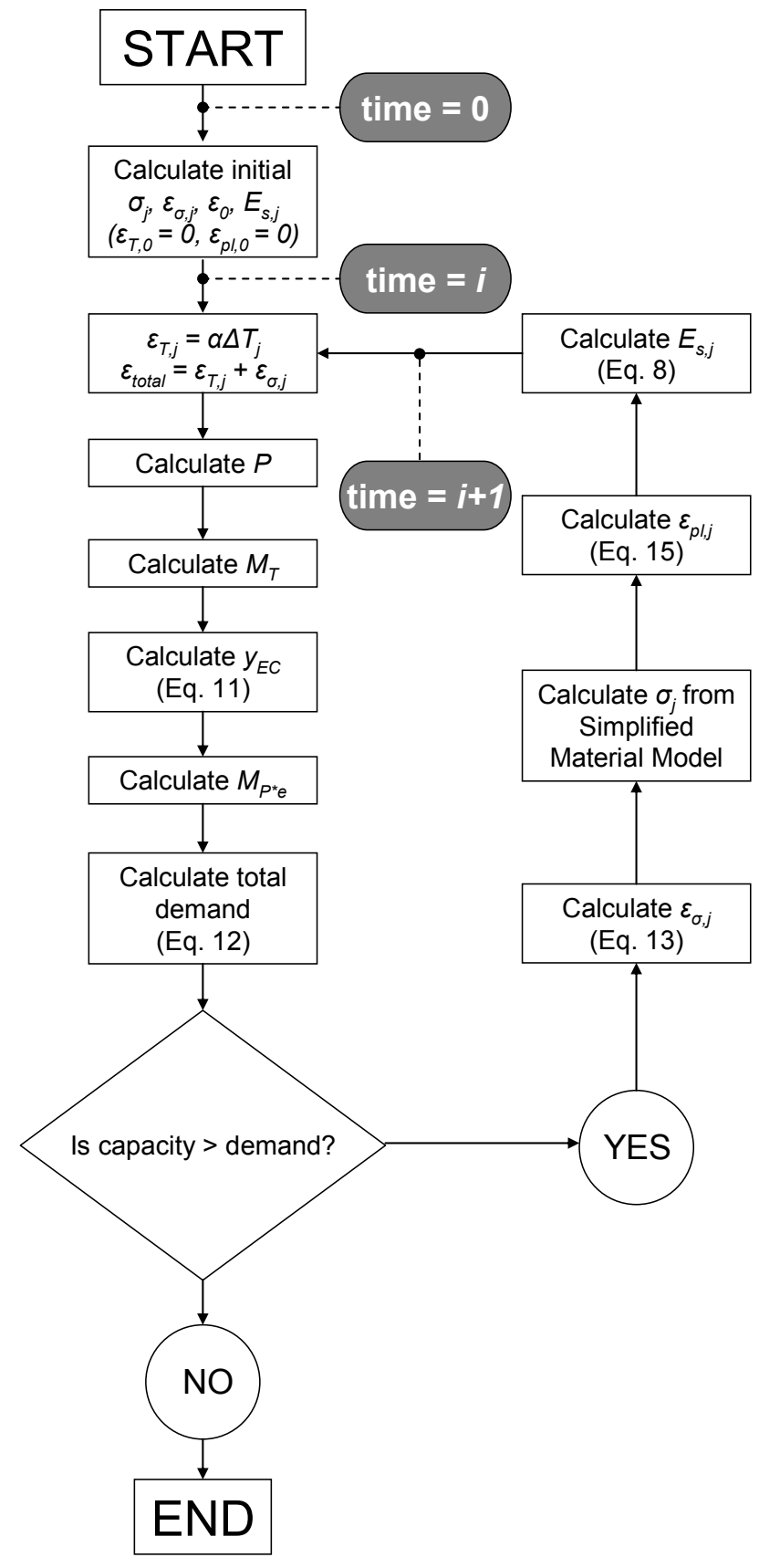

Fig. (11). Step-by-step procedure for simplified structural analysis.

lar expression as that shown in [2], $y_{E C}$ is calculated at every time step as follows:

$$
y_{E C}=\eta \frac{\sum_{j} E_{s, j} y_{j} A_{j}}{\sum_{j} E_{s, j} A_{j}}
$$

Similar to Eq. 10, the adjustment factor $\eta=0.75$ is added to this expression to account for the overestimation of strain and underestimation of $E_{s, j}$ by the simplified material model. Once the effective centroid no longer coincides with the geometric centroid, axial loads applied at the geometric centroid (as is the case with the loads considered for this study) will generate a moment, $M_{P^{*} e}$, equal to $P$ times $y_{E C}$. By using the geometric centroid as the reference axis, the calculation for $P^{*} y_{E C}$ at every time step using Eq. 11 will yield a nonzero value only when the effective centroid does not coincide with the geometric centroid.

\section{Step-by-Step Procedure}

Fig. (11) shows a flowchart describing the step-by-step procedure to perform the simplified calculation of demand. The material model corresponding to the temperature at the current time step $i$ (with corresponding temperaturedependent material reduction factors based on Eurocode [5]) is constructed as shown in Fig. (10) and is used to make this calculation. To initialize the procedure, it is assumed that the perimeter column is cool and loaded in its elastic range with initially applied axial load $P$. Initial values of $\sigma_{j}, \varepsilon_{\sigma, j}$, and $E_{s, j}$ in each coarse fiber are therefore calculated with elastic material properties and the initial $P$. Thermal strains $\left(\varepsilon_{T, j}\right)$ and plastic strain $\left(\varepsilon_{p l, j}\right)$ are zero (as shown in Fig. (11) in the box following time $=0)$.

At the beginning of every time step $i$, values for $\sigma_{j}, \varepsilon_{\sigma, j}$, and $E_{s, j}$ are obtained from time step $i$ - 1 . Thermal strains $\varepsilon_{T, j}$ are calculated according to Eurocode [5] based on the current fiber temperatures at time step $i$, and $\varepsilon_{\text {total }}$ (which is constant for all fibers) is calculated as the sum of $\varepsilon_{T, j}$ and $\varepsilon_{\sigma, j} . P, M_{T}$, and $M_{P *_{e}}$ collectively represent the total demand on the beam-column and are calculated using the simplified expressions outlined in the previous three subsections. The $P$ and total $M$ experienced by the member at time step $i$ are then normalized by their respective values of plastic capacity $\left(P_{y}\right.$ and $M_{p}$ ) at that time step:

$$
\left(\frac{M_{T}+M_{P^{*} e}}{M_{p}}, \frac{P}{P_{y}}\right)_{(i)}
$$

Plotting these normalized $P-M$ combinations for each time step generates a "path" of structural behavior. At each time step, the point calculated in Eq. 12 is compared against the plastic $P-M$ capacity envelope to check for full section yielding. If the point calculated using Eq. 12 falls outside the plastic $P-M$ capacity envelope (constructed using Eqs. 6 and 7 ), then the section has fully yielded. A check can also made at this point to make sure that the cross-section has not exceeded its buckling strength. If the section's capacity is greater than its demand, then failure has not been reached and the analysis continues.

For the next time step $i+1$, updated values of $\sigma_{j}, \varepsilon_{\sigma, j}$, and $E_{s, j}$ are calculated based on the combined effects of $P, M_{T}$, and $M_{P^{*} e}$ at time step $i$, as shown in Fig. (11). The stressrelated strain $\left(\varepsilon_{\sigma, j}\right)$ in each fiber $j$ is calculated first as follows:

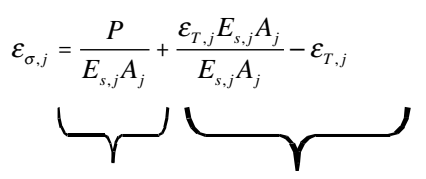

due to $\mathrm{P}$ due to $M_{T}+M_{P * e}$

Note that the values of $E_{s, j}$ used for this calculation, which were also used to calculate $P, M_{T}$, and $M_{P^{*}{ }^{*}}$, still correspond to time step $i$.

Fig. (12) traces the evolution of stress and strain in fiber $j$ from time step $i$ to time step $i+1$ assuming that the fiber had 


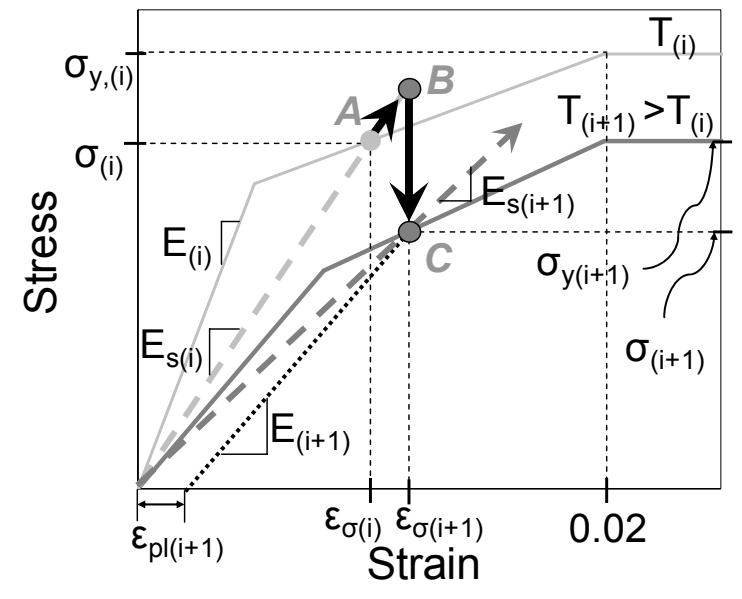

Fig. (12). Simplified material models for increasing temperature, showing plastic strain and secant stiffness.

been previously loaded to point $\boldsymbol{A}$. During time step $i+1$, the fiber is loaded from point $\boldsymbol{A}$ to point $\boldsymbol{B}$ via the secant stiffness $E_{s}$ corresponding to time step $i$. Having solved for $\varepsilon_{\sigma, j}$ at time step $i+1$ via Eq. 13, the geometry of the simplified material model can be used to calculate the corresponding value of $\sigma_{j}$ for the time step $i+l$ (point $\boldsymbol{C}$ ). Once this calculation is made, the plastic strain in every fiber is calculated to update the geometry of the simplified material model. An approximate calculation for $\varepsilon_{p l}$ for fiber $j$ at time step $i$ is made using the geometry shown in Fig. (12):

$$
\varepsilon_{p l, j}=\left(\varepsilon_{\sigma, j}-\frac{\sigma_{j}}{E_{j}}\right)
$$

where $E_{j}$ is the elastic modulus for fiber $j$ at time step $i+1$ $\left(E_{20} k_{E, T, j(i+1)}\right)$. The secant stiffness $E_{s, j}$ (represented in Fig. (12) as the slope of the line connecting point $C$ to the origin) corresponds to the updated values of $\sigma_{j}$ and $\varepsilon_{\sigma, j}$ and is calculated according to Eq. 8. Using the updated values of $\sigma_{j,} \varepsilon_{\sigma, j}$, and $E_{s, j}$, the procedure to calculate demand is repeated for the next time step as shown in Fig. (11). A maximum time step length of 60 seconds is recommended to ensure that the transition between time steps is calculated at sufficiently small values of strain.

\section{VALIDATION STUDIES}

The ability of the simplified thermal and structural analysis methodologies to accurately predict the response of perimeter columns exposed to fire is validated via comparison to the results of computational FE analysis. The results of the experimental study discussed in [3] have also been successfully used to validate these methodologies; however, these comparisons are not shown here since it would add considerable length to this paper to do so. The thermal and structural responses shown by the computational FE models discussed in this study have also been validated using the experimental results presented in [3].

Two prototypes representing perimeter columns will be discussed in this paper: 1MP-S and 1MP-W. Both columns $\left(F_{y, 20}=250 \mathrm{MPa}\right.$; initial $\left.P / P_{y}=25 \%\right)$ are based on actual perimeter columns from the One Meridian Plaza (1MP) steel building frame, which suffered a severe fire in 1991 and has been studied by the authors [20]. Column 1MP-S experi-

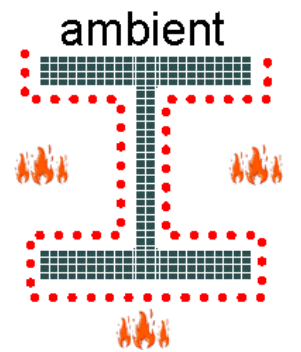

(a)

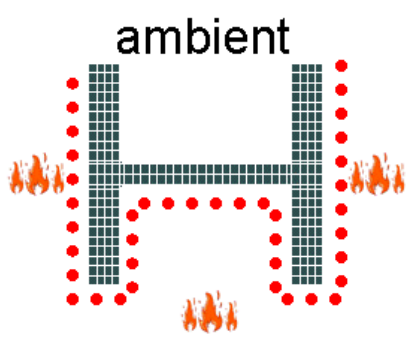

(b)
Fig. (13). Fire exposed cross-sections: (a) 1MP-S and (b) 1MP-W.

ences thermal gradients (and thus bending) in the strong axis direction, and 1MP-W experiences thermal gradients and bending in the weak axis direction. Columns 1MP-S and $1 \mathrm{MP}-\mathrm{W}$ represent an idealized, isolated $\mathrm{W} 14 \times 314$ perimeter column in the fire-exposed portion of the 1MP building frame. These columns are assumed to have no applied fire protection and are exposed on three sides to fire. The discretized cross-sections used for thermal and structural FE analysis of 1MP-S and 1MP-W, including the orientation of the three-sided fire exposure, are shown in Fig. (13(a) and (b)). The temperature-time curve used for this study, shown in Figs. (14(a) and (b)), was developed previously by the authors to approximate the 1MP fire [20]. Validation of the simplified thermal analysis methodology for sections with applied fire protection is provided by Quiel [3].

Computational thermal and structural analysis of each case was performed using SAFIR, a software specifically designed for the analysis of structures exposed to fire [24]. Thermal and structural properties for steel used for computational analysis were according to Eurocode [5]. Each case was also analyzed using the simplified thermal and structural analysis methodologies outlined above. The closed-form simplified methodology was implemented using Matlab, a widely available mathematical analysis software, but a spreadsheet could also have been used. The Matlab programs written by the authors to implement the simplified member model are provided in [3]. A 60-second time step was used for both the computational and simplified analyses.

The convective heat transfer coefficient, $h$, and relative emissivity, $\varepsilon$, for the fire-exposed surfaces were assumed to be $25 \mathrm{~W} / \mathrm{m}^{2}-\mathrm{K}$ and 0.5 , respectively, to simulate the heat transfer of a compartment fire [15]. For non-exposed surfaces, $h$ was modeled with a value of $9 \mathrm{~W} / \mathrm{m}^{2}-\mathrm{K}$ to account for reduced convection in the absence of hot gases. Computational thermal analyses of these cross-sections produced realistic temperature distributions similar to those shown in Figs. (2(b) and $\mathbf{3}(\mathbf{b}))$. These temperatures were then used as input for the structural analysis model shown in Fig. $(\mathbf{8}(\mathbf{a}))$, where the same cross-sectional temperature distribution was input along the column's entire length.

Both the FE and simplified structural models of the 1MP columns consider the loading and boundary conditions shown in Fig. (8(a)) with $L=3.56 \mathrm{~m}$. FE modes used threenoded beam elements, discretized to $50-\mathrm{mm}$ lengths, whose cross-sections were discretized into numerous fibers. These fiber-beam elements are capable of representing full yield and global buckling limit states. The vertical spring is as- 
(a)

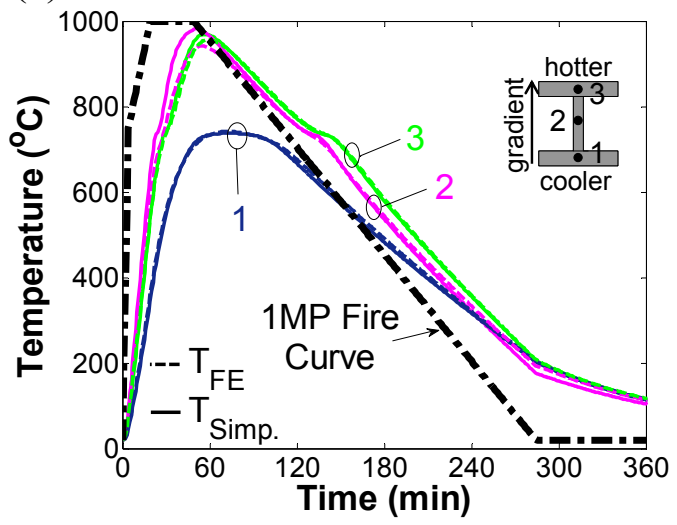

(b)

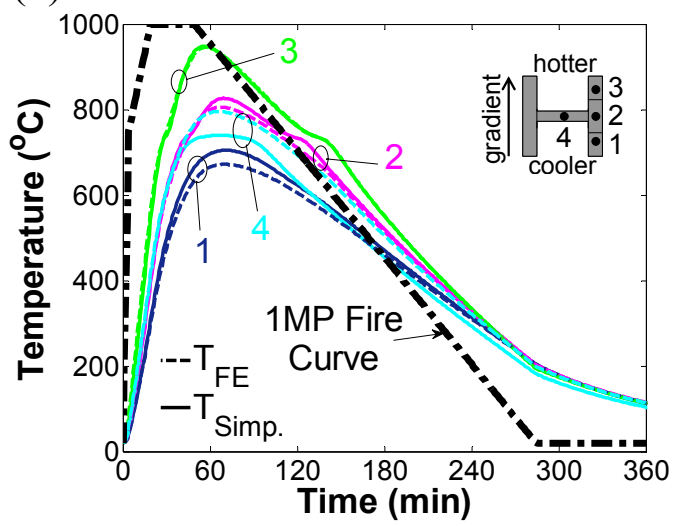

Fig. (14). Cross-sectional temperature comparisons (a) 1MP-S and (b) 1MP-W.

sumed to have zero stiffness since the adjacent structure is not considered, and the columns are modeled with a constant axial load equal to $25 \%$ of initial $P_{y}$. Structural FE analysis of these cases provides a direct validation of the simplified structural analysis methodology because both use the same loading and boundary conditions. Validation of perimeter column behavior for models whose vertical spring has nonzero stiffness is provided by Quiel [3].

\section{Validating the Simplified Thermal Analysis Methodology}

The fire-exposed cross-sections in Figs. (2(a) and 3(a)) are used to validate the ability of the simplified thermal analysis methodology to calculate the temperature in each coarse fiber. The temperature of the lumped masses in the $1 \mathrm{MP}-\mathrm{S}$ and $1 \mathrm{MP}-\mathrm{W}$ cross-sections is calculated using Eqs. 1 and 2, respectively, because their three-sided fire exposure (see Figs. 13(a) and (b)) matches the fire exposure shown in Figs. (4 and 5) for which the equations were formulated. Since the sections are unprotected, $Q_{\text {in }}$ and $Q_{\text {out }}$ are calculated according to Eq. 3 using the same values for $h$ and $\varepsilon$ as the computational analyses. Thermal properties for steel are according to Eurocode [5].

In Figs. (14(a) and (b)), temperatures calculated for each fiber using the simplified thermal methodology $\left(\mathrm{T}_{\text {Simp }}\right)$ are compared to the average temperature in each fiber calculated from thermal $\mathrm{FE}$ analysis of the $1 \mathrm{MP}$ sections $\left(\mathrm{T}_{\mathrm{FE}}\right)$ for the two validation cases. These plots show that the simplified thermal analysis provides a good prediction of the nonuniform temperature distribution that develops through the cross-sectional depth.

\section{Validating the Simplified Structural Analysis Methodol- ogy}

Fig. (1) illustrates the solutions used to validate the ability of the simplified structural analysis methodology to predict the capacity and demand of perimeter columns exposed to fire (i.e. a steel beam-column that develops a thru-depth thermal gradients). The Pure FE solution is calculated for the $1 \mathrm{MP}$ validation cases as a baseline against which the simplified structural solutions for the 1MP columns are compared. The $\mathrm{T}_{\mathrm{FE}} \mathrm{S}_{\text {Simp }}$ solution is used to validate the simplified structural analyses for both validation cases, respectively, so that the same thermal input is used for both the simplified and FE

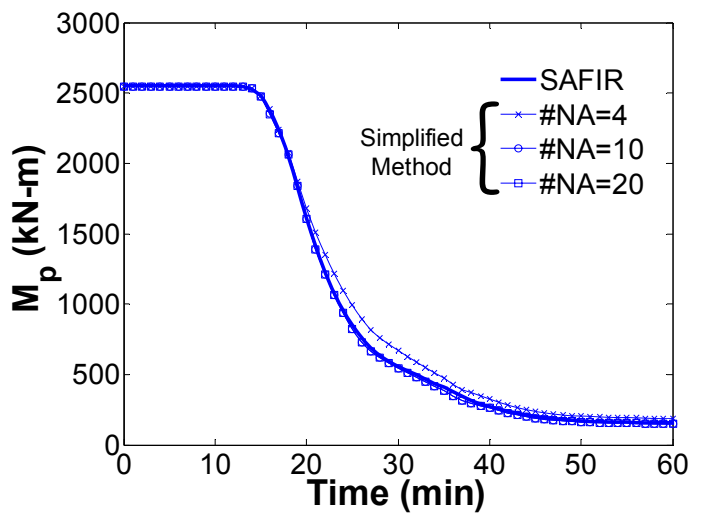

Fig. (15). Plot of $M$ for $1 \mathrm{MP}-\mathrm{S}$.

structural analyses. This allows a comparison of the two structural analyses with the prediction of temperature removed as a variable.

\section{Combined P-M Yield Capacity}

As discussed previously in this paper, it is recommended that Eqs. 6 and 7 be solved at least at ten incremental NA locations through the depth of the section to obtain accurate estimates for plastic moment, $M_{p}$, and the shape of the plastic $P-M$ capacity envelope when using the simplified prediction of capacity. Fig. (15) shows a comparison of the values of $M_{p}$ calculated for the $1 \mathrm{MP}-\mathrm{S}$ cross-section during the first hour of fire exposure using Eqs. 6 and 7 with different numbers of NA locations. Also shown is the curve of $M_{p}$ predicted via FE analysis of the highly discretized cross-section shown in Fig. (13(a)). It is shown that using only four NA positions provides a slightly unconservative estimate of $M_{p}$ compared to the FE prediction. There is little difference between the FE prediction and the simplified prediction corresponding to ten and twenty NA locations, and therefore using ten discrete NA locations provides adequate accuracy. The other three validation cases show similar results in this regard.

Fig. (16) shows a plot of the plastic $P-M$ capacity envelopes predicted by the FE models and the simplified structural analysis methodology for heated cross-sections of both validation cases at various times during fire exposure. The 
(a)

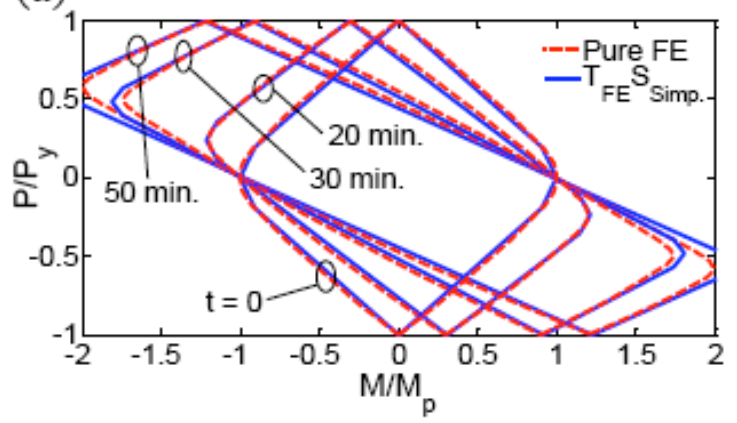

(b)

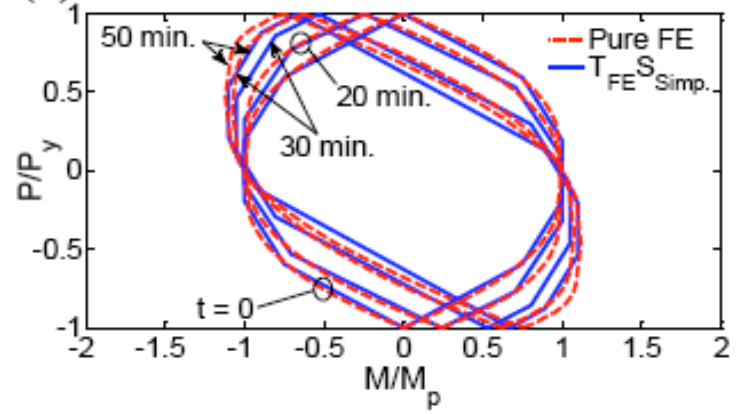

Fig. (16). Plots of plastic $P-M$ capacity envelopes for (a) $1 \mathrm{MP}-\mathrm{S}$ and (b) $1 \mathrm{MP}-\mathrm{W}$ at various times during fire exposure.

(a)

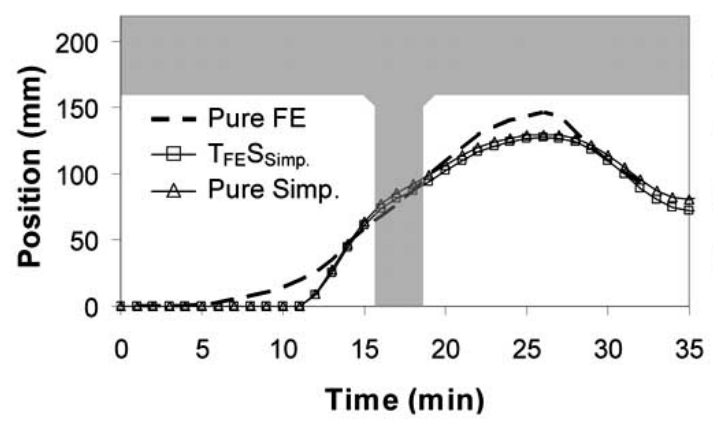

Fig. (17). Plot of $y_{E C}$ for (a) $1 \mathrm{MP}-\mathrm{S}$ and (b) 1MP-W.

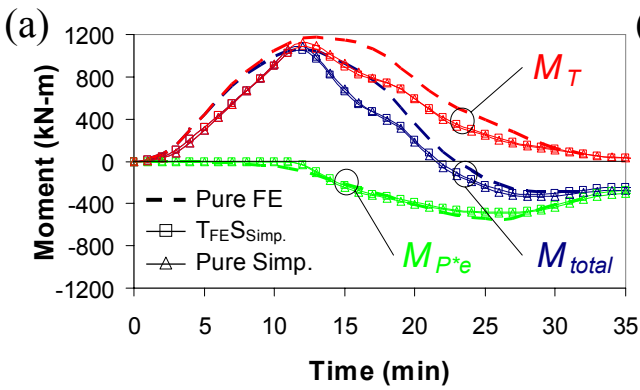

(b)

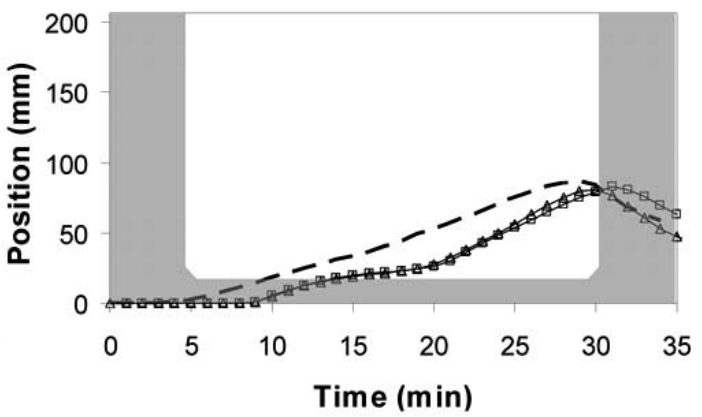

(b)

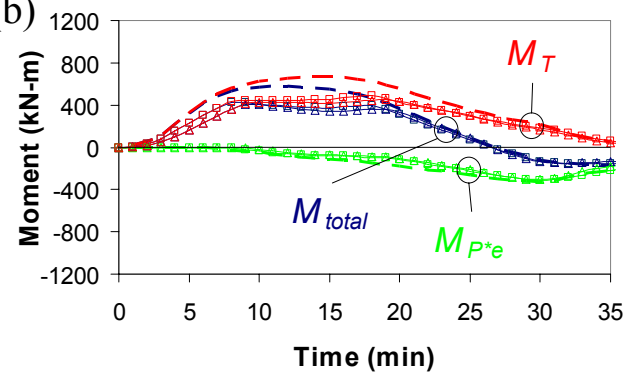

Fig. (18). Plot of moment components for (a) 1MP-S and (b) 1MP-W.

computationally derived capacity envelopes marked "Pure FE" were derived using the methods developed by the authors [1] for thermal profiles obtained via FE thermal analysis of the discretized cross-sections shown in Fig. (13). The envelopes corresponding to simplified structural analysis solutions $\left(\mathrm{T}_{\mathrm{FE}} \mathrm{S}_{\mathrm{Simp}}\right.$ ) were constructed according to the method outlined previously using the coarse-fiber thermal profiles. The plots in Fig. (16) show close agreement between the FE and simplified predictions of plastic capacity.

\section{Combined P-M Demand}

Figs. (17(a) and (b)) show the position of the effective centroid $\left(y_{E C}\right)$ for columns $1 \mathrm{MP}-\mathrm{S}$ and $1 \mathrm{MP}-\mathrm{W}$, respectively, calculated via FE and simplified structural analysis. The shaded region in each plot represents the cooler half of the wide-flanged cross-section and is used to illustrate the position of $y_{E C}$ relative to the section's depth. Initially, the effective centroid coincides with the geometric centroid (at position $=0$ ) and then migrates into the cooler half of the section due to the thermal gradient. The FE and simplified structural analyses provide similar predictions of $y_{E C}$. Figs. $(\mathbf{1 8}(\mathbf{a})$ and (b)) show plots of the total moment, $M_{\text {total }}$, experienced by each 1MP column during fire exposure as well as its two components, $M_{T}$ and $M_{P^{*} e}$. Initially, the columns develop positive $M_{T}$ because the rotationally rigid boundary conditions restrict thermal bowing, and $M_{\text {total }}$ equals $M_{T}$ since $y_{E C}$ still coincides with the geometric centroid. When $y_{E C}$ migrates toward the cooler half of the section, negative $M_{P^{*} e}$ is generated. At this time, $M_{\text {total }}$ peaks and begins to reverse direction as the rate of increase of $M_{P^{*} e}$ becomes larger than the rate of increase of $M_{T}$. Eventually, $M_{P^{*} e}$ becomes larger than $M_{T}$, and $M_{\text {total }}$ reverses sign. Good agreement is again shown between the FE and simplified predictions.

\section{Predicting Failure}

Fig. (19) shows plots of $P$ and $M$ for the FE and simplified structural analyses of each validation case at the time of failure. The values of $P$ and $M$ are normalized by their corre- 

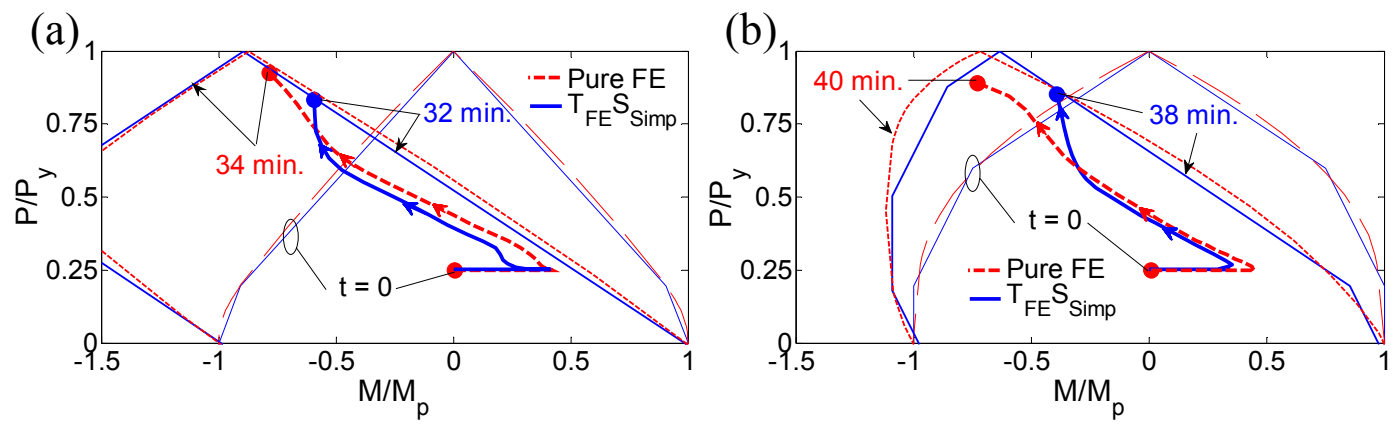

Fig. (19). Plots of normalized $P-M$ performance for (a) $1 \mathrm{MP}-\mathrm{S}$ and (b) $1 \mathrm{MP}-\mathrm{W}$.
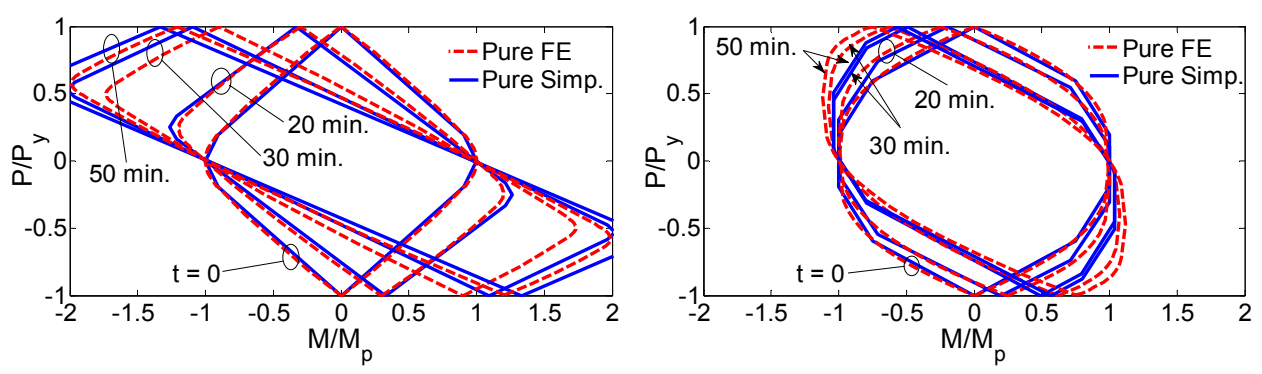

Fig. (20). Plots of plastic $P-M$ capacity envelopes for (a) $1 \mathrm{MP}-\mathrm{S}$ and (b) $1 \mathrm{MP}-\mathrm{W}$ at various times during fire exposure.

sponding yield strength $\left(P_{y}\right)$ and plastic moment $\left(M_{p}\right)$ at every time step according to Eq. 12. Each plot also includes the normalized plastic $P-M$ capacity envelopes for $t=0$ (i.e just prior to fire exposure) and for the temperature distribution at the time of failure. Since moment is constant along the length of the column (Fig. 8), the plotted path of $P$ and $M$ therefore describes the ratio of demand to capacity for the entire column.

The path of $P-M$ combinations during fire exposure progresses from the point marked $t=0$ to a point marked with a specific time of failure. The $P-M$ behavior of both validation cases in Fig. (19) shows good agreement between the FE and simplified structural analyses. The plots of normalized $P-M$ for all analyses show both the initial increase in positive moment $\left(M_{T}\right)$ and the moment reversal due to increasing negative values of $M_{P^{*} \text { e }}$. The significant increase in axial load ratio $P / P_{y}$ for each case was caused primarily by a decrease in $P_{y}$. The magnitude of $M / M_{p}$ was also amplified by a simultaneous decrease in plastic capacity. The coupled effects of increasing load and decreasing capacity push the section closer to its plastic capacity until the path of normalized $P$ and $M$ reaches the capacity envelope and analysis ends, indicating that the section has fully yielded.

Table 1 summarizes the times at which full section yield occurs in the FE analyses using fiber-beam elements in SAFIR and the solution obtained using the simplified struc-

Table 1 - Recorded times (in minutes) to column failure.

\begin{tabular}{||cccc||}
\hline Case & Pure FE & $\mathbf{T}_{\mathrm{FE}} \mathbf{S}_{\text {Simp }}$ & $\begin{array}{c}\text { Pure } \\
\text { Simplified }\end{array}$ \\
\hline \hline 1MP-S & 34 & 32 & 32 \\
$1 \mathrm{MP}-\mathrm{W}$ & 40 & 38 & 37 \\
\hline
\end{tabular}

tural analysis methodology. The FE and simplified structural analyses show good agreement in predicting the time at which each column experienced an intersection of capacity and demand as illustrated in Fig. (19), and the simplified structural analyses consistently provided conservative predictions of performance. This agreement, which is a product of the close agreement between the individual predictions of capacity and demand, confirms that the closed-form simplified structural analysis methodology can effectively predict the changes in capacity and demand experienced by perimeter columns under fire (which develop a thru-depth thermal gradient and act as beam-columns).

\section{Validating the Combination of Simplified Thermal and Structural Analysis}

The results of simplified thermal analysis can be used to construct the coarse-fiber temperature profile needed as input for simplified structural analysis. This combination, (i.e. the Pure Simplified solution in Fig. 1) provides a closed-form prediction of the total response of a perimeter column to fire exposure. The Pure Simplified solutions for both validation cases use the temperatures marked $\mathrm{T}_{\text {Simp }}$ in Fig. (14) as input for simplified structural analysis. The results of these simplified analyses are compared to the solutions using FE structural analysis (Pure FE) to validate the accuracy of the combined thermal and structural simplified analysis methodology.

Fig. (20) shows reasonable agreement of the plastic $P-M$ capacity envelopes predicted by the Pure Simplified solution and the FE structural analyses for both validation cases at several times during fire exposure. Variation between the simplified and computational/experimental temperatures (see Fig. 14) translates into slightly more variation in the shape of the plastic $P-M$ envelopes in Fig. (20) as compared to Fig. (16) (i.e. the $\mathrm{T}_{\mathrm{FE}} \mathrm{S}_{\mathrm{Simp}}$ solutions). Figs. (17 and 18) show that the predictions of demand are less sensitive to variations in 

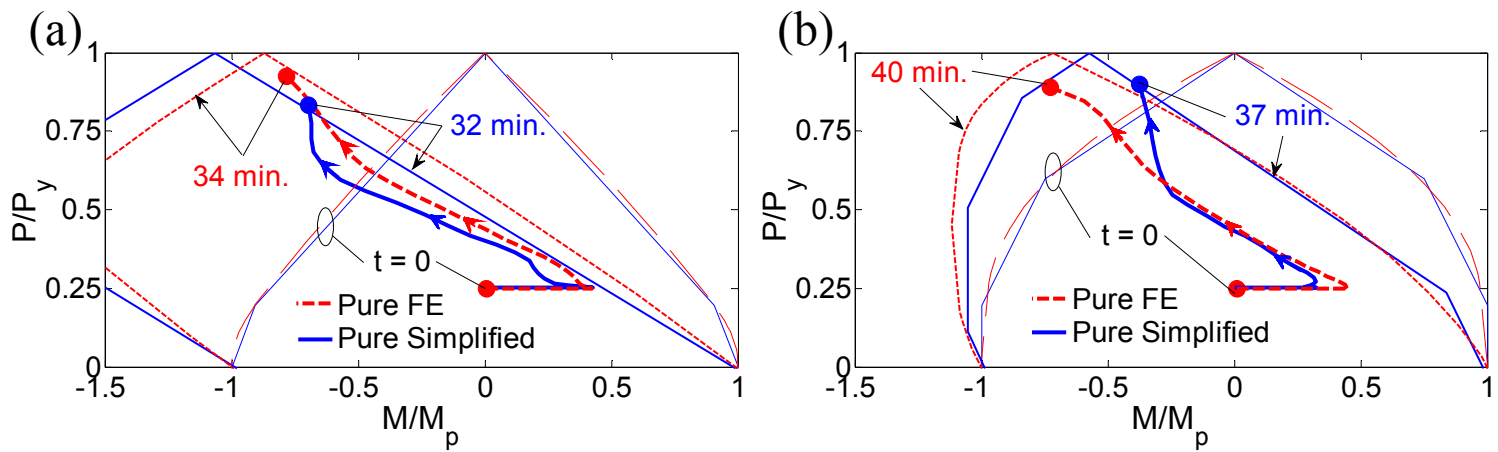

Fig. (21). Plots of normalized $P-M$ performance for (a) $1 \mathrm{MP}-\mathrm{S}$ and (b) $1 \mathrm{MP}-\mathrm{W}$.

predicted temperature because all solutions using simplified structural analysis (i.e. the $\mathrm{T}_{\mathrm{FE}} \mathrm{S}_{\mathrm{Simp}}$ and Pure Simplified solutions) are similar and agree well with those using FE structural analysis (Pure FE). Fig. (21) shows the time and pathway with which the normalized $P-M$ points reach the plastic capacity envelope show good agreement between the Pure Simplified solutions and those predicted via FE structural analysis. Table 1 shows that the analyses all predicted similar times to failure. These comparisons demonstrate that the combination of the simplified thermal and structural analyses can be used to accurately calculate the fire-exposed response of steel perimeter column using only closed-form expressions.

\section{SUMMARY AND CONCLUSIONS}

A simplified methodology is proposed to calculate the response of steel beam-columns that develop a thermal gradient through their depth due to non-uniform fire exposure. The current version of the methodology focuses on the performance of wide-flanged perimeter columns under fire, although it could be adapted for the analysis of other sections shapes or beam-column cases (such as floor beams). The expressions developed for simplified time-series integration are closed-form, allowing the user to explicitly calculate thermal and structural response at each time step without using iterative or computational methods. The proposed methodology, referred to as the simplified member model, has three primary components, each of which was outlined in detail in this paper: (1) a simplified thermal analysis, (2) a simplified prediction of capacity, and (3) a simplified prediction of demand. Together, components (2) and (3) comprise a simplified structural analysis. Both the simplified thermal and structural analyses subdivide the cross-section of the beam-column into a small number of coarse fibers. Based on previous work by Ghojel and Wong [16], heat transfer between these fibers (represented as lumped masses) and the surrounding environment can be calculated to obtain a nonuniform temperature distribution about either the strong or weak axis. The capacity and demand of the column is calculated via summation of the strength, stress, and strain of each fiber in response to temperature and loading and is compared at every time step. The results of these analyses can be combined to provide a closed-form prediction of the total response of a perimeter column under fire.

Two perimeter column prototypes were used to validate our simplified thermal and structural analysis methodologies against computational results. These methodologies were also validated against experimental results [3], although not discussed in this paper. Coarse-fiber temperatures calculated for each validation case via simplified thermal analysis showed good agreement with the results of FE thermal analysis. The simplified structural analyses (with thermal FE inputs) provided similar predictions of capacity and demand, as well as the mode and time to failure, as the FE models. Both the thermal and structural simplified methodologies were combined to obtain a pure simplified solution that did not rely on any FE analyses. These pure simplified solutions also showed good agreement with the results of FE structural analysis.

The simplified methodologies proposed in this paper offer a simple, closed-form alternative to computational FE analysis to calculate the response of perimeter columns to fire. The good agreement of simplified predictions with computational and experimental results indicates that simple methods can be used to effectively and efficiently predict the response of beam-columns that develop thermal gradients through their depth. The simplified methodology can be implemented in a spreadsheet or other non-iterative mathematical algorithm and has potential as a useful tool for the performance-based design of steel beam-columns under fire. The closed-form calculations presented in this paper can be incorporated into a performance-based design procedure for steel perimeter columns, which is currently being developed by the authors [3].

\section{ACKNOWLEDGEMENTS}

The research presented in this paper is based on work that is co-sponsored by the National Science Foundation (NSF) (under grant number CMMI-0652282) and the National Institute of Standards and Technology (NIST) (under grant number 60NANB7D6121). Dr. Quiel's involvement with this research project began while on appointment as a U.S. Department of Homeland Security (DHS) Fellow under the DHS Scholarship and Fellowship Program, which is administered by the Oak Ridge Institute for Science and Education (ORISE) for DHS through an interagency agreement with the U.S Department of Energy (DOE). ORISE is managed by Oak Ridge Associated Universities under DOE contract number DE-AC05-00OR22750. All opinions, findings, and conclusions expressed in this dissertation are the authors' and do not necessarily reflect the policies and views of the DHS, DOE, ORISE, NSF, or NIST.

\section{REFERENCES}

[1] M. E. M Garlock and S. E. Quiel, "Plastic axial load - moment interaction curves for fire-exposed steel sections", Journal of Structural Engineering (ASCE), vol. 134, pp. 874-880, 2008. 
[2] M. E. M. Garlock, and S. E. Quiel, "Mechanics of wide-flanged steel sections that develop thermal gradients due to fire exposure", International Journal of Steel Structures (KSSC), vol. 7, pp. 153$162,2007$.

[3] S. E. Quiel, "Behavior and Analysis of Fire-Exposed Steel BeamColumns that Develop Thermal Gradients", PhD Thesis, Princeton University: Princeton, NJ, USA, June 1, 2009.

[4] R. G. Gann, ed., NIST NCSTAR 1: Final Report of the National Construction Safety Team on the Collapse of the World Trade Center Twin Towers, Gaithersburg, MD: National Institute of Standards and Technology (NIST), 2005.

[5] CEN, Eurocode 3: Design of Steel Structures, Part 1.2: General Rules - Structural Fire Design (ENV 1993-1-2:2001), Brussels: European Committee for Standardization (CEN), 2001.

[6] AISC, Steel Construction Manual, $13^{\text {th }}$ Ed., Chicago: American Institute of Steel Construction (AISC), 2005.

[7] J. Takagi and G. G. Deierlein, "Strength design criteria for steel members at elevated temperatures", Journal of Constructional Steel Research, vol. 63, pp. 1036-1050, 2007.

[8] M. Knobloch, M. Fontana and A. Frangi, "Steel beam-columns subjected to fire", Steel Construction, vol. 1, pp. 51-58, 2008.

[9] N. Lopes, L. Simões da Silva, P. M. M. Vila Real and P. Piloto, "New proposals for the design of steel beam-columns in case of fire, including a new approach for the lateral torsional buckling", Computers and Structures, vol. 82, pp. 1463-1472, 2004.

[10] W. Skowronski, "Buckling fire endurance of steel columns", Journal of Structural Engineering (ASCE), vol. 119, pp. 1712-1732, 1993.

[11] Z. F. Huang and K. H. Tan, "Analytical fire resistance of axially restrained steel columns", Journal of Structural Engineering (ASCE), vol. 129, pp. 1531-1537, 2003.

[12] G. Q. Li, W. Y. Wang and S. W. Chen, "A simple approach for modeling fire-resistance of steel columns with locally damaged fire protection", Engineering Structures, vol. 31, pp. 617-622, 2009.
[13] A. S. Usmani, J. M. Rotter, S. Lamont, A. M. Sanad and M. Gillie, "Fundamental principles of structural behaviour under thermal effects", Fire Safety Journal, vol. 36, pp. 721-744, 2001.

[14] W. L. Gamble, "Predicting protected steel member fire endurance using spread-sheet programs", Fire Technology, vol. 25, pp. 256$273,1989$.

[15] A. H. Buchanan, Structural Design for Fire Safety, Chichester, UK: John Wiley \& Sons, 2002.

[16] J. I. Ghojel and M. B. Wong, "Three-sided heated of I-beams in composite construction exposed to fire", Journal of Constructional Steel Research, vol. 61, pp. 834-844, 2005.

[17] CEN, Eurocode 3: Design of Steel Structures, Part 1.5: Plated Structural Elements (ENV 1993-1-5:2004), Brussels: European Committee for Standardization (CEN), 2004.

[18] M. Knobloch and M. Fontana. "Strain-based approach to local buckling of steel sections subjected to fire", Journal of Constructional Steel Research, vol. 62, pp. 44-67, 2006.

[19] P. M. M. Vila Real, N. Lopes, L. Simões da Silva, P. Piloto and J. M. Franssen, "Numerical modelling of steel beam-columns in case of fire - comparisons with Eurocode 3", Fire Safety Journal, vol. 39, pp. 23-39, 2004.

[20] M. E. M. Garlock and S. E. Quiel, "The behavior of steel perimeter columns in a high-rise building under fire", Engineering Journal (AISC), vol. 44, pp. 359-372, 2007.

[21] J. M. Franssen, "The unloading of building materials submitted to fire," Fire Safety Journal, vol. 16, pp. 213-227, 2009.

[22] J. A. El-Rimawi, I. W. Burgess and R. J. Plank, "The analysis of semi-rigid frames in fire - a secant approach", Journal of Constructional Steel Research, vol. 33, pp. 125-146, 1995.

[23] I. W. Burgess, J. A. El-Rimawi and R. J. Plank, "Analysis of beams with non-uniform temperature profile due to fire exposure", Jour nal of Constructional Steel Research, vol. 16, pp. 169-192, 1990.

[24] J. M. Franssen, "SAFIR: A thermal/structural program for modeling structures under fire", Engineering Journal (AISC), vol. 42, pp. $143-158,2005$

Received: June 25, 2009

(C) Quiel and Garlock; Licensee Bentham Open.

This is an open access article licensed under the terms of the Creative Commons Attribution Non-Commercial License (http://creativecommons.org/licenses/by-nc/3.0/) which permits unrestricted, non-commercial use, distribution and reproduction in any medium, provided the work is properly cited. 